\title{
DNA methylation: a new twist in the tail
}

\author{
Gavin Kelsey ${ }^{1,2}$ \\ ${ }^{I}$ Epigenetics Programme, The Babraham Institute, Cambridge, CB22 3AT; ${ }^{2}$ Centre for Trophoblast Research, University of Cam- \\ bridge, Cambridge, CB2 3EG, United Kingdom \\ Cell Research (2011) 21:1155-1156. doi:10.1038/cr.2011.110; published online 5 July 2011
}

DNA methylation is the epigenetic mark with the longest history and that we probably understand best, yet we still have no adequate account for why specific DNA sequences are selected to become methylated. Gene-specific DNA methylation is fundamental to processes such as developmental silencing of genes, classical epigenetic phenomena such as genomic imprinting, and occurs pathologically in the silencing of tumor suppressor genes in cancer. Fully understanding the mechanisms of methylation is thus of huge importance. In mammals, the acquisition of DNA methylation is determined by one of two de novo DNA methyltransferase enzymes, Dnmt3a and Dnmt3b. These activities are assisted by the related, but catalytically inactive protein Dnmt3L. Dnmt3a and Dnmt3b have similar structures, comprising a PWWP domain, PHD-like or ADD domain and a carboxy-terminal catalytic domain [1]. The PWWP domains are required for binding of Dnmt3a and Dnmt3b to chromatin in vivo, and the PHD domain is thought to be dispensable for this [2]. Dnmt3L, which is a truncated protein lacking the PWWP and a functional catalytic domain, forms a heterotetramer with Dnmt3a or Dnmt3b [3] and is described as stimulating the activity of its de novo

Correspoindence: Gavin Kelsey

Tel: 01223 496332; Fax: 01223496022

E-mail: gavin.kelsey@babraham.ac.uk partners, or guiding the recognition of DNA targets with a particular periodicity of $\mathrm{CpG}$ sites [3], or attenuating the inherent sequence specificities of Dn$\mathrm{mt} 3 \mathrm{a}$ or Dnmt3b so that they methylate targets more homogenously [4].

It has long been suspected that there would be cross-talk between DNA methylation and chromatin state, and a key observation came four years ago when it was shown that the PHD domain of Dnmt3L interacts with the animoterminal tail of histone $\mathrm{H} 3$, but that specific methylation of the lysine at residue 4 (H3K4) inhibits this interaction [5]. This made a good deal of sense, as $\mathrm{H} 3 \mathrm{~K} 4$ methylation is a marker for active genes and there is an inverse correlation between the presence of trimethylated H3K 4 and DNA methylation at promoters. Moreover, the H3K4 demethylase $\mathrm{KDM} 1 \mathrm{~B}$ is required for establishment of DNA methylation at the control regions of a subset of imprinted genes [6]. Subsequently, similar properties of the PHD domains of Dnmt3a and Dnmt3b have been described [7]; in addition, the PWWP domain of Dnmt3a specifically recognizes trimethylated $\mathrm{H} 3 \mathrm{~K} 36$ [8]. Together, these observations suggest that an appropriate combination of histone modifications serves as a docking platform for the de novo methylation complexes. The study by $\mathrm{Li}$ and colleagues [9] published recently in Cell Research adds an interesting new twist to the mechanism by which histone modifications might contribute to de novo DNA methylation.

The authors found that amino-terminal peptides of $\mathrm{H} 3$ not only bound the PHD domain of Dnmt3a, but also actually stimulated its activity in vitro. This effect was sensitive to the modification state of lysine 4, as stimulation was progressively reduced with increasing methylation and abolished by trimethylation. To demonstrate the in vivo significance of this observation, Dnmt3a carrying point mutations in residues essential for $\mathrm{H} 3$ tail binding, but not affecting basal enzyme activity in vitro, were stably transfected into mouse ES cells lacking endogenous Dnmt3a and Dnmt $3 b$ and were shown to be incapable of methylating the Oct4 promoter upon differentiation, although binding to Oct4 was apparently not affected. These initial results were interpreted to suggest that binding of the $\mathrm{H} 3$ tail causes allosteric activation of Dnmt3a. To investigate this possibility further, the authors mutated residues predicted to be involved in the interfaces between the PHD and catalytic domains of Dnmt3a. Two residues were identified at which substitutions strongly impaired the ability of $\mathrm{H} 3$ peptides to stimulate Dnmt3a activity in vitro, again without affecting basal activity. These mutations were then tested in the in vivo setting. Importantly, neither mutation altered the ability of Dnmt3a to complex with Dnmt3L, or its binding to the Oct4 promoter or major satellite DNA but, again, the ability of both mutants to 
methylate these target sequences was blocked, strengthening the conclusion that the interaction of $\mathrm{H} 3$ tails with the PHD domain of Dnmt3a is required for allosteric activation. From these results, the authors present a novel, two-step model for methylation by Dnmt3a in which the protein is recruited to chromatin via its PWWP domain, or through interactions with Dnmt3L or other chromatin-bound proteins, but is activated only by binding of the PHD domain by unmethylated $\mathrm{H} 3 \mathrm{~K} 4$.

Does this interesting new twist help us to understand how specific DNA sequences are targeted for methylation? One challenge is how to incorporate these observations into a model that includes Dnmt3a in its association with Dnmt3L. In vivo it appears that Dnmt3a depends largely on Dnmt3L, as revealed by genome-wide analysis of $\mathrm{CpG}$ island methylation in mouse oocytes [10]. The Dnmt3a:Dnmt3L heterotetramer seems to be a complex bristling with recognition sites receptive to histone tail modifications, and these might act synergistically (on single or adjacent nucleosomes?) so that at preferred genomic targets all sites are engaged with the de novo complex with ensuing stimulation of Dnmt3a. Is H3 tail binding to the PHD domain of Dnmt3L, which itself lacks catalytic function, communicated within the complex to further enhance the activity of Dnmt3a? Or does the interaction of Dnmt3L with unmodified $\mathrm{H} 3$ tails serve primarily to help specify binding of the complex, as Dnmt3L seems to have a major influence on the subnuclear localization of Dnmt3a [11]? We might need more sophisticated assays to identify targets of Dnmt3a and their chromatin properties, and whether choice of target is modified by Dnmt3L. In any case, the study emphasizes the critical role of histone tail modification status in determining binding and activity of the de novo methylation complex.

\section{References}

1 Cheng X, Blumenthal RM. Mammalian DNA methyltransferases: a structural perspective. Structure 2008; 16:341350.

2 Ge YZ, Pu MT, Gowher H, et al. Chromatin targeting of de novo DNA methyltransferases by the PWWP domain. $J$ Biol Chem 2004; 279:25447-25454.

3 Jia D, Jurkowska RZ, Zhang X, Jeltsch A, Cheng X. Structure of Dnmt3a bound to Dnmt3L suggests a model for de novo DNA methylation. Nature 2007; 449:248-251.

4 Wienholz BL, Kareta MS, Moarefi AH, et al. DNMT3L modulates significant and distinct flanking sequence preference for DNA methylation by DN-
MT3A and DNMT3B in vivo. PLoS Genet 2010; 6: e1001106.

5 Ooi SK, Qiu C, Bernstein E, et al. DNMT3L connects unmethylated lysine 4 of histone $\mathrm{H} 3$ to de novo methylation of DNA. Nature 2007; 448:714-717.

6 Ciccone DN, Su H, Hevi S, et al. KD$\mathrm{M} 1 \mathrm{~B}$ is a histone $\mathrm{H} 3 \mathrm{~K} 4$ demethylase required to establish maternal genomic imprints. Nature 2009; 461:415-418.

7 Zhang Y, Jurkowska R, Soeroes S, et al. Chromatin methylation activity of Dnmt3a and Dnmt3a/3L is guided by interaction of the ADD domain with the histone H3 tail. Nucl Acids Res 2010; 38:4246-4253.

8 Dhayalan A, Rajavelu A, Rathert P, et al. The Dnmt3a PWWP domain reads histone 3 lysine 36 trimethylation and guides DNA methylation. $J$ Biol Chem 2010; 285:26114-26120.

9 Li BZ, Huang Z, Cui QY, et al. Histone tails regulate DNA methylation by allosterically activating de novo methyltransferase. Cell Res 2011; 21:11721181.

10 Smallwood SA, Tomizawa S, Krueger $\mathrm{F}$, et al. Dynamic CpG island methylation landscape in oocytes and preimplantation embryos. Nat Genet 2011 Jun 26. doi:10.1038/ng.864

11 Jurkowska RZ, Rajavelu A, Anspach N, et al. Oligomerization and binding of the DNMT3A DNA methyltransferase to parallel DNA molecules, heterochromatic localization and role of DNMT3L. J Biol Chem 2011; 286:2420024207 